\title{
HIERARCHY AMONG AUTOMATA ON LINEAR ORDERINGS
}

\author{
Véronique Bruyère \\ Institut d'Informatique, Université de Mons-Hainaut, \\ Le Pentagone, 6 avenue du Champ de Mars, \\ B-7000 Mons, Belgium \\ Veronique.Bruyere@umh.ac.be \\ Olivier Carton \\ Institut Gaspard Monge, Université de Marne-la-Vallée, \\ 5 boulevard Descartes, \\ F-77454 Marne-la-Vallée Cedex 2, France \\ Olivier.Carton@univ-mlv.fr
}

\begin{abstract}
In a preceding paper, automata and rational expressions have been introduced for words indexed by linear orderings, together with a Kleenelike theorem. We here pursue this work by proposing a hierarchy among the rational sets. Each class of the hierarchy is defined by a subset of the rational operations that can be used. We then characterize any class by an appropriate class of automata, leading to a Kleene theorem inside the class. A characterization by particular classes of orderings is also given.
\end{abstract}

\section{Introduction}

The first result in automata theory and formal languages is the Kleene theorem which establishes the equivalence between sets of words accepted by automata and sets of words described by rational expressions. Since the seminal paper of Kleene [8], this equivalence has been extended to many kinds of structures: infinite words, bi-infinite words, finite and infinite trees, finite and infinite traces, pictures, etc.

In [3], we have considered linear structures in a general framework, i.e., words indexed by a linear ordering. This approach allows to treat in the same way finite words, left- and right-infinite words, bi-infinite words, ordinal words which are studied separately in the literature. We have introduced a new notion of automaton accepting words on linear orderings, which is simple, natural and includes previously defined automata. We have also defined rational expressions 
for such words. We have proved the related Kleene-like theorem when the orderings are restricted to countable scattered linear orderings. This result extends Kleene's theorem for finite words [8], infinite words [4, 10], bi-infinite words $[7,11]$ and ordinal words $[5,6,16]$.

Another jewel of formal languages is the characterization of star-free languages by first-order logic [9] or by group-free semigroups [14]. A set of finite words is star-free if it can be described by a rational expression using concatenation, union and complementation only. The class of star-free sets is thus obtained by restricting the rational operations. The star iteration is replaced by complementation which is weaker when union and concatenation are already allowed.

In this paper, we propose a hierarchy among rational sets of words on linear orderings. As for star-free sets, this hierarchy is obtained by restricting the rational operations that can be used. Each class contains the rational sets that can be described by a given subset of the rational operations.

The rational operations introduced in [3] include the usual operations of union, concatenation and star iteration. They also include the omega iteration usually used to construct infinite words and the ordinal iteration introduced by Wojciechowski [16] for ordinal words. Three new operations are added: the backwards omega iteration, the backwards ordinal iteration and a last operation which is a kind of iteration for all countable scattered linear orderings. The lowest class of the hierarchy contains sets that can be described by rational expressions using union, concatenation and star iteration. This is of course the class of rational sets of finite words. The greatest class contains sets that can be described by rational expressions using all rational operations introduced in [3]. It contains all rational sets of words on scattered linear orderings. Some other classes of words already studied in the literature appear naturally in our framework. Sets of words on ordinals introduced by Büchi [5] or sets of words on ordinals smaller that $\omega^{\omega}$ studied by Choueka [6] form two classes of our hierarchy.

We give a characterization of each class of the hierarchy by a corresponding class of automata. A set of words belongs to the given class if and only if it is recognized by an automaton of the corresponding class. Each of these characterizations is thus a Kleene theorem which holds for that class. For wellknown classes, these Kleene theorems were already proved by Wojciechowski [16] for words on ordinals or by Choueka [6] for words on ordinals smaller than $\omega^{\omega}$. In each case, the corresponding class of automata is obtained naturally by restricting the kind of transitions that can be used. For instance, the automata for words on ordinals do have left limit transitions but no right limit transitions as there were defined by Büchi [5].

The last rational operation defined in [3] works like an iteration for all countable scattered linear orderings. It is binary. In this paper, we consider a simpler definition of this iteration as a unary operation. This simplified definition seems to be more natural but it turns out to be weaker. The results of this paper 
show that the binary operation is really needed to obtain the Kleene theorem of [3]. This question was actually the original motivation of our work.

We also give a characterization of each class of the hierarchy by a corresponding class of orderings. A set of words belongs to the given class if and only if the length of each of its words belongs to the corresponding class of orderings. For some classes as the class of sets of words on ordinals, this characterization is straightforward. However for some other classes, suitable classes of orderings have to be defined. These definitions are inspired by the characterization of countable scattered orderings due to Hausdorff.

To summarize, the results of the paper establish a hierarchy among rational sets of words on linear orderings, with connections between natural classes of orderings, rational operations and the types of transitions in automata.

The paper is organized as follows. In Sections 2, 3 and 4, we briefly recall the new notions introduced in [3]: words on linear orderings, automata and rational expressions. We refer the reader to [13] for a complete introduction to linear orderings. The different classes of the hierarchy are described in Section 5. This hierarchy is summarized on Figure 6 and illustrated by some examples. The proofs are given in the Appendix.

\section{Orderings and Words}

A linear ordering $J$ is an ordering < which is total, that is, for any $j \neq k$ in $J$, either $j<k$ or $k<j$ holds. Given a finite alphabet $A$, a word $\left(a_{j}\right)_{j \in J}$ is a function from $J$ to $A$ which maps any element $j$ of $J$ to a letter $a_{j}$ of $A$. We say that $J$ is the length $|x|$ of the word $x$. For instance, the empty word $\varepsilon$ is indexed by the empty linear ordering $J=\varnothing$. Usual finite words are the words indexed by finite orderings $J=\{1,2, \ldots, n\}, n \geq 0$. A word of length $J=\omega$ is a word usually called an $\omega$-word or an infinite word. A word of length $J=\zeta$ is a sequence $\ldots a_{-2} a_{-1} a_{0} a_{1} a_{2} \ldots$ of letters which is usually called a bi-infinite word.

Given a linear ordering $J$, we denote by $-J$ the backwards linear ordering obtained by reversing the ordering relation. For instance, $-\omega$ is the backwards linear ordering of $\omega$ which is used to indexed the so-called left-infinite words. For a class $\mathcal{V}$ of linear orderings, we denote by $-\mathcal{V}$ the class $\{-J \mid J \in \mathcal{V}\}$.

Given two linear orderings $J$ and $K$, the linear ordering $J+K$ is obtained by juxtaposition of $J$ and $K$, i.e., it is the linear ordering on the disjoint union $J \cup K$ extended with $j<k$ for any $j \in J$ and any $k \in K$. For instance, the linear ordering $\zeta$ can be obtained as the sum $-\omega+\omega$. More generally, let $J$ and $K_{j}$ for $j \in J$, be linear orderings. The linear ordering $\sum_{j \in J} K_{j}$ is obtained by juxtaposition of the orderings $K_{j}$ in respect of $J$. More formally, the sum $\sum_{j \in J} K_{j}$ is the set $L$ of all pairs $(k, j)$ such that $k \in K_{j}$. The relation $\left(k_{1}, j_{1}\right)<\left(k_{2}, j_{2}\right)$ holds iff $j_{1}<j_{2}$ or $j_{1}=j_{2}$ and $k_{1}<k_{2}$ in $K_{j_{1}}$.

The sum operation on linear orderings leads to a notion of product of words as follows. Let $J$ and $K_{j}$ for $j \in J$, be linear orderings. Let $x_{j}=\left(a_{k, j}\right)_{k \in K_{j}}$ be a word of length $K_{j}$, for any $j \in J$. The product $\prod_{j \in J} x_{j}$ is the word $z$ of length $L=\sum_{j \in J} K_{j}$ equal to $\left(a_{k, j}\right)_{(k, j) \in L}$. For instance, the word $a^{\zeta}=a^{-\omega} \cdot a^{\omega}$ of 
length $\zeta=-\omega+\omega$ is the product of the two words $a^{-\omega}$ and $a^{\omega}$ of length $-\omega$ and $\omega$ respectively.

In this paper as in [3], we only consider linear orderings which are countable and scattered, i.e., without any dense subordering. This class is denoted by $\mathcal{S}$ and its elements are shortly called orderings. We use notation $\mathcal{N}$ for the subclass of $\mathcal{S}$ of finite linear orderings and $\mathcal{O}$ for the subclass of countable ordinals. Recall that an ordinal is a linear ordering which is well-ordered, that is, without the subordering $-\omega$.

The following characterization of the class $\mathcal{S}$ is due to Hausdorff [13]. Notation 1 is used for the finite ordering with one element and notation $\beta<\alpha$ means the usual ordering on ordinals.

Theorem 1 (Hausdorff) $\mathcal{S}=\bigcup_{\alpha \in \mathcal{O}} U_{\alpha}$ where the classes $U_{\alpha}$ are inductively defined by

$$
\begin{aligned}
& 1 U_{0}=\{\varnothing, 1\} \\
& 2 U_{\alpha}=\left\{\sum_{j \in J} K_{j} \mid J \in \mathcal{N} \cup\{\omega,-\omega, \zeta\} \text { and } K_{j} \in \bigcup_{\beta<\alpha} U_{\beta}\right\} .
\end{aligned}
$$

For instance, the ordinal $\omega$ belongs to $U_{1}$ because $\omega=\sum_{j \in \omega} K_{j}$ with $K_{j}=1 \epsilon$ $U_{0}$. More generally one can check that the ordinal $\omega^{n}$ belongs to $U_{n} \backslash U_{n-1}$, for all $n \geq 1$. Finally, $\omega^{\omega}$ belongs to $U_{\omega}$ since it equals $\sum_{j \in \omega} K_{j}$ with $K_{j}=\omega^{j} \in$ $U_{j}$, but it belongs to no $U_{n}$ (see Chapter 5 of [13]).

We propose two new families of classes to characterize $\mathcal{S}$. The class $\mathcal{S}$ is equal to $\bigcup_{\alpha \in \mathcal{O}} V_{\alpha}$ where the classes $V_{\alpha}$ are inductively defined by

$1 V_{0}=\{\varnothing, 1\}$

$2 V_{\alpha}=\left\{\sum_{j \in J} K_{j} \mid J \in \mathcal{O} \cup\{-\omega, \zeta\}\right.$ and $\left.K_{j} \in \bigcup_{\beta<\alpha} V_{\beta}\right\}$.

It is also equal to $\bigcup_{\alpha \in \mathcal{O}} W_{\alpha}$ where the classes $W_{\alpha}$ are inductively defined by

$1 W_{0}=\{\varnothing, 1\}$

$2 W_{\alpha}=\left\{\sum_{j \in J} K_{j} \mid J \in \mathcal{O} \cup-\mathcal{O}\right.$ and $\left.K_{j} \in \bigcup_{\beta<\alpha} W_{\beta}\right\}$.

The ordinal $\omega^{\omega}$ belongs to $V_{1}$ and $W_{1}$. The backwards ordinal $-\omega^{\omega}$ belongs to $V_{\omega}$ and to $W_{1}$. More generally, $\mathcal{O} \subseteq V_{1}$ and $\mathcal{O} \cup-\mathcal{O} \subseteq W_{1}$. It can be proved that the ordering $\zeta^{\omega}$ (see [13] for a precise definition) belongs to $W_{\omega}$ but not to $\bigcup_{n<\omega} W_{n}$.

\section{Automata}

Automata accepting words on linear orderings are a natural extension of finite automata. As above they are defined as $\mathcal{A}=(Q, A, E, I, F)$. The set $E$ is composed with three types of transitions: the usual successor transitions in $Q \times A \times E$, the left limit transitions which belong to $\mathcal{P}(Q) \times Q$ and the right limit transitions which belong to $Q \times \mathcal{P}(Q)$. 


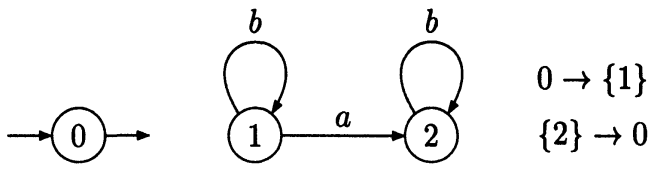

Figure 1. An automaton on linear orderings

Example 1 The automaton depicted in Figure 1 has one left limit transition $\{2\} \rightarrow 0$ and one right limit transition $0 \rightarrow\{1\}$.

The notion of cut is needed to define a path in such an automaton. A cut of an ordering $J$ is a pair $(K, L)$ of intervals such that $J=K \cup L$ and for any $k \in K$ and $l \in L, k<l$. The two subsets must be disjoint and they form a partition of the set $J$. The set of all cuts of the ordering $J$ is denoted by $\hat{J}$. The set $\hat{J}$ can be linearly ordered as follows. For any cuts $c_{1}=\left(K_{1}, L_{1}\right)$ and $c_{2}=\left(K_{2}, L_{2}\right)$, define the relation $c_{1}<c_{2}$ iff $K_{1} \subsetneq K_{2}$. Note that $\hat{J}$ has always a least cut $(\varnothing, J)$ denoted $c_{\min }$ and a greatest cut $(J, \varnothing)$ denoted $c_{\max }$.

See Figure 2 where each element of $J$ is represented by a bullet, and each cut by a vertical bar.

$$
|\cdots| \cdot|\cdot| \cdot|\cdots| \cdots|\cdot| \cdot|\cdot| \cdots \mid
$$

Figure 2. Ordering $J \cup \hat{J}$ for $J=\zeta+\zeta$.

A word $x=\left(a_{j}\right)_{j \in J}$ of length $J$ is accepted by $\mathcal{A}$ if it is the label of a successful path. A path $\gamma$ is a sequence of states $\gamma=\left(q_{c}\right)_{c \in \hat{J}}$ of length $\hat{J}$ verifying the following conditions. For two consecutive states in $\gamma$, there must be a successor transition labeled by the letter in between. For a state $q \in \gamma$ which has no predecessor on $\gamma$, there must be a left limit transition $P \rightarrow q$ where $P$ is the limit set of $\gamma$ on the left of $q$. Right limit transitions are used similarly when $q$ has no successor on $\gamma$. A path is successful if its first state $q_{c_{\min }}$ is initial and its last state $q_{c_{\max }}$ is final.

More precisely, for any cut $c \in \hat{J}$, define the sets $\lim _{c^{-}} \gamma$ and $\lim _{c^{+}} \gamma$ as follows:

$$
\begin{aligned}
& \lim _{c^{-}} \gamma=\left\{q \in Q \mid \forall c^{\prime}<c \exists k \quad c^{\prime}<k<c \text { and } q=q_{k}\right\}, \\
& \lim _{c^{+}} \gamma=\left\{q \in Q \mid \forall c<c^{\prime} \exists k \quad c<k<c^{\prime} \text { and } q=q_{k}\right\} .
\end{aligned}
$$

For any consecutive cuts $c_{j}^{-}$and $c_{j}^{+}$of $\hat{J}, q_{c_{j}^{-}} \stackrel{a_{j}}{\longrightarrow} q_{c_{j}^{+}}$must be a successor transition. For any cut $c \neq c_{\min }$ in $\hat{J}$ which has no predecessor, $\lim _{c^{-}} \gamma \rightarrow q_{c}$ 
must be a left limit transition. For any cut $c \neq c_{\min }$ in $\hat{J}$ which has no successor, $q_{c} \rightarrow \lim _{c^{+}} \gamma$ must be a right limit transition.

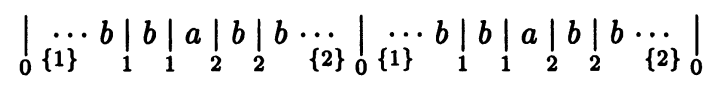

Figure 3. The word $\left(b^{-\omega} a b^{\omega}\right)^{2}$ is accepted

The notion of path $\gamma$ we have introduced for words on orderings coincide with the usual notion of paths considered in the literature for finite words [12], $\omega$-words [15] and ordinal words [2]. For a Muller automaton accepting $\omega$-words, a left limit set $P$ is computed at the end of the path. It is nothing else than the states appearing infinitely often along the path. In our context, the path then ends with an additional left limit transition to a state $q$ which is final.

\section{Rational Expressions}

We now introduce the notion of rational sets of words on linear orderings. The rational operations of course include the usual Kleene operations for finite words which are the union + , the concatenation - and the star operation $*$. They also include the omega iteration $\omega$ usually used to construct $\omega$-words and the ordinal iteration $\sharp$ introduced by Wojciechowski [16] for ordinal words. Three new operations are also needed: the backwards omega iteration $-\omega$, the backwards ordinal iteration $-\sharp$ and a last binary operation denoted $\diamond$ which is a kind of iteration for all orderings.

Given two sets $X$ and $Y$ of words, we define

$$
\begin{aligned}
X+Y & =\{z \mid z \in X \cup Y\} \\
X \cdot Y & =\{x \cdot y \mid x \in X, y \in Y\} \\
X^{*} & =\left\{\prod_{j \in\{1, \ldots, n\}} x_{j} \mid n \in \mathcal{N}, x_{j} \in X\right\} \\
X^{\omega} & =\left\{\prod_{j \in \omega} x_{j} \mid x_{j} \in X\right\} \\
X^{-\omega} & =\left\{\prod_{j \in-\omega} x_{j} \mid x_{j} \in X\right\} \\
X^{\sharp} & =\left\{\prod_{j \in \alpha} x_{j} \mid \alpha \in \mathcal{O}, x_{j} \in X\right\} \\
X^{-\sharp} & =\left\{\prod_{j \in-\alpha} x_{j} \mid \alpha \in \mathcal{O}, x_{j} \in X\right\} \\
X \diamond Y & =\left\{\prod_{j \in J \cup j^{*}} z_{j} \mid J \in \mathcal{S}, z_{j} \in X \text { if } j \in J \text { and } z_{j} \in Y \text { if } j \in \hat{J}^{*}\right\} .
\end{aligned}
$$

The last operation needs some explanation. Notation $\hat{J}^{*}$ is used for the set $\hat{J} \backslash\{(\varnothing, J),(J, \varnothing)\}$. A word $x$ belongs to $X \diamond Y$ iff there is an ordering $J$ such that $x$ is the product indexed by the ordering $J \cup \hat{J}^{*}$ of words where each word indexed by an element of $J$ belongs to $X$ and each word indexed by a cut in $\hat{J}^{*}$ belongs to $Y$ (see Figure 4). We use notation $X^{\diamond}$ for the set $X \diamond \varepsilon$. When operation $\diamond$ is used as a unary operation, we also use notation $\diamond_{1}$. When it is used as a binary operation, we use notation $\diamond_{2}$. 


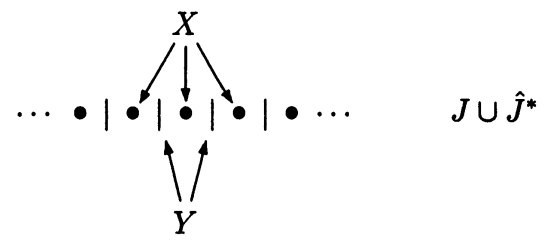

Figure 4. The operation $X \diamond Y$

Note that the definitions for the operation $*$ and $\sharp$ are closed to each other. The only difference is that the products are over any $J \in \mathcal{N}$ for operation * whereas they are over any $J \in \mathcal{O}$ for operation $\sharp$.

Example 2 The set of all words over the alphabet $A$ is the rational set $A^{\diamond}=$ $A \diamond \varepsilon$. The set of words accepted by the automaton of Figure 1 is the rational set $\left(b^{-\omega} a b^{\omega}\right)^{*}$.

In [3], we show that a set of words on countable scattered linear orderings is accepted by an automaton iff it can be described by a rational expression. This result extends the well-known Kleene theorem on finite words, its extension to $\omega$-words [4] and to ordinal words [16].

Theorem 2 ([3]) Over countable scattered linear orderings, $X \subseteq A^{\circ}$ is recognizable iff it is rational.

The proof that any rational set of words is recognizable is by induction on the rational expression denoting the set by giving the corresponding construction for the automaton. The constructions for the union, the concatenation and the star iteration are very similar to the classical ones for automata on finite words [12]. The proof that any set of words accepted by an automaton is rational is a generalization of McNaughton and Yamada algorithm. It is based on a induction on the number of states of the automaton and the type of transition that is used. The base of the induction is Kleene's theorem on finite and $\omega$-words. This part of the proof is the most difficult.

Example 3 The automaton pictured in Figure 5 accepts the set denoted by the rational expression $a^{\zeta} \diamond b$. The part of the automaton given by state 2 and the two limit transitions $0 \rightarrow\{2\}$ and $\{2\} \rightarrow 1$ accepts the word $a^{\zeta}$ whereas the part given by the successor transition from state 1 to state 0 accepts the word $b$. Any occurrence of $a^{\zeta}$ is preceded and followed by an occurrence of $b$ in the automaton. Thanks to the limit transitions $0 \rightarrow\{0,1,2\}$ and $\{0,1,2\} \rightarrow 1$, the occurrences of $a^{\zeta}$ are indexed by an ordering $J$, the occurrences of $b$ are indexed by the ordering $\hat{J}^{*}$ and they are interleaved according to the ordering $J \cup \hat{J}^{*}$. 


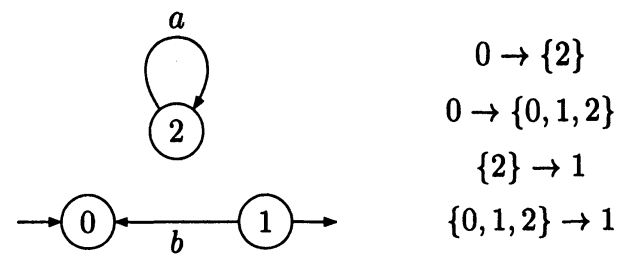

Figure 5. Automaton accepting the set $a^{\zeta} \diamond b$

\section{Hierarchy}

We now come to the main result of this paper. We introduce a hierarchy among rational sets of words on countable scattered linear orderings. Each class of this hierarchy is obtained by restricting the rational operations that can be used. It turns out that each of these classes can be characterized by automata with a particular form. These classes of automata are defined by restricting the limit transitions of the automata. In other words, a Kleene-like theorem holds for each class of the hierarchy. Finally, each class of rational sets of our hierarchy can be characterized by a class of orderings. For any rational set of a given class, the lengths of all its words belong to the corresponding class of orderings.

For instance, rational sets with operations restricted to,$+ \cdot$ and $*$ correspond to automata without any limit transitions. The corresponding orderings are the finite ones.

The different classes of the hierarchy are summarized on Figure 6. Let us give a precise description of each class.

0 This class corresponds to Kleene theorem [8] on rational sets of finite words. The rational operations are the usual Kleene operations: union, concatenation and star iteration. The automata are the usual automata on finite words with no limit transitions. The orderings are the finite ones.

1 This class corresponds to Choueka theorem [6] on rational sets of words of length an ordinal smaller than $\omega^{\omega}$. The rational operations are the Kleene ones and the omega iteration. Automata considered by Choueka are a special kind of automata on ordinals introduced by Büchi. It can be shown [1] that these automata are equivalent to automata with no right limit transitions and such that the left limit transitions are of the form $P \rightarrow q$ with $q \notin P$. The corresponding orderings are the ordinals smaller than $\omega^{\omega}$.

2 The rational operations of Class 2 are the Kleene ones, the omega iteration and the backwards omega iteration. The automata have left limit transitions $P \rightarrow q$ with $q \notin P$ and right limit transitions $q^{\prime} \rightarrow P^{\prime}$ 


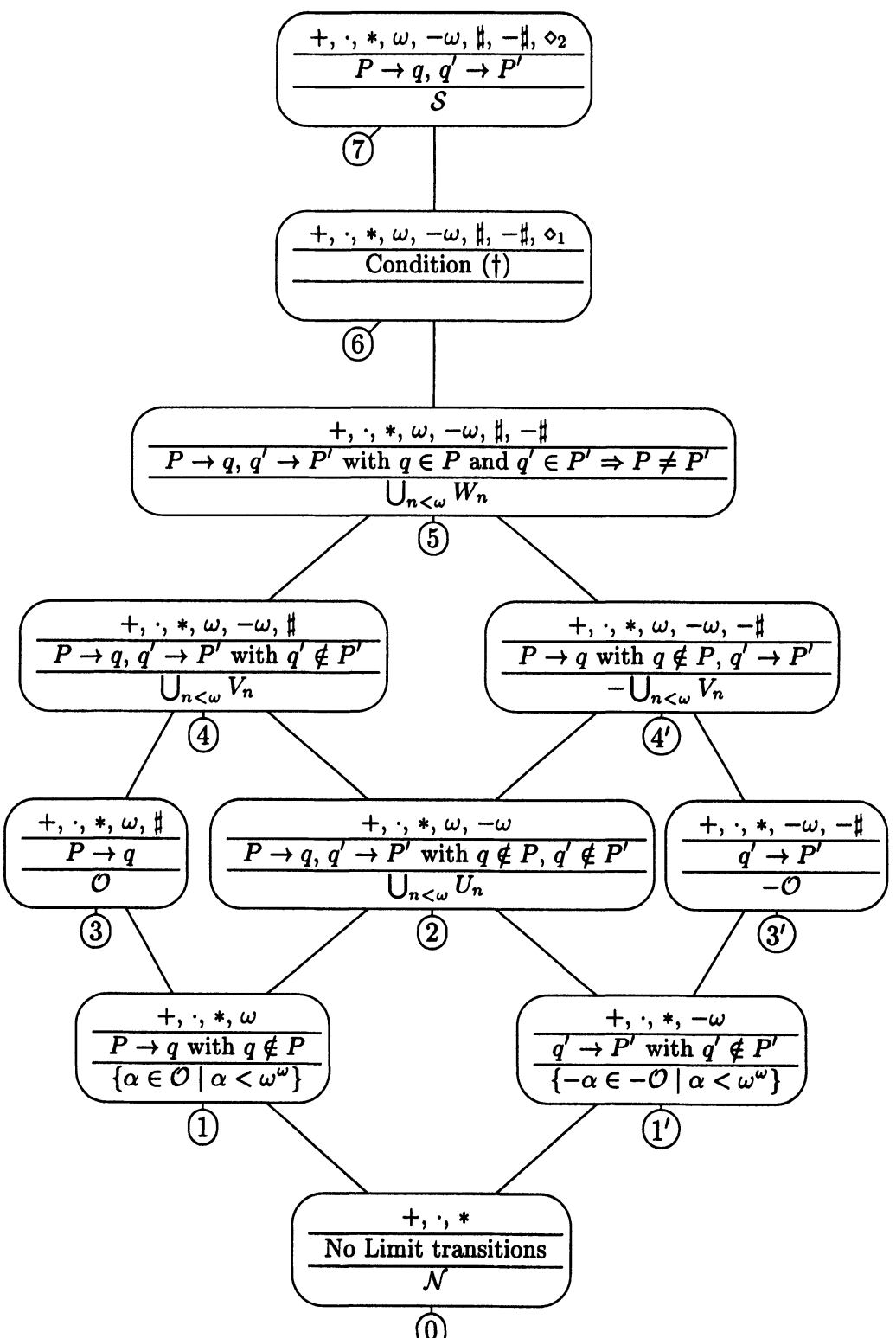

Figure 6. Classes of the hierarchy 
with $q^{\prime} \notin P^{\prime}$. The associated orderings are the orderings belonging to $\bigcup_{n<\omega} U_{n}$ where the classes $U_{n}$ have been defined in Theorem 1.

3 This class corresponds to Wojciechowski theorem [16] on rational sets of words on ordinals. The rational operations are the Kleene ones, the omega iteration and the ordinal iteration introduced by Wojciechowski. The automata are those on ordinals introduced by Büchi [5]. They have any left limit transitions of the form $P \rightarrow q$ but they have no right limit transitions. The related orderings are exactly those of the class $\mathcal{O}$ of all countable ordinals.

4 The rational operations of Class 4 are the Kleene one, the omega iteration, the backwards omega iteration and the ordinal iteration. The automata have any left limit transitions $P \rightarrow q$ but right limit transitions $q^{\prime} \rightarrow P^{\prime}$ limited by the condition $q^{\prime} \notin P^{\prime}$. The associated orderings are the orderings belonging to $\bigcup_{n<\omega} V_{n}$ where the classes $V_{n}$ have been defined just after Theorem 1.

5 In this class, all rational operations are allowed except the operation $\diamond$. The automata have left and right limit transitions $P \rightarrow q$ and $P^{\prime} \rightarrow q^{\prime}$ restricted by the following condition: if $q \in P$ and $q^{\prime} \in P^{\prime}$, then one has $P \neq P^{\prime}$. The related class of orderings is equal to $\bigcup_{n<\omega} W_{n}$ (see the definition of $W_{n}$ just after Theorem 1).

6 In this class, all rational operations are allowed but the operation $\diamond$ can only be used as a unary operation, that is the operation $X \diamond Y$ must be restricted to the case $Y=\{\varepsilon\}$. The automata have left and right limit transitions $P \rightarrow q$ and $q^{\prime} \rightarrow P^{\prime}$ of a particular form.

Condition ( $\dagger$ ). Let $P \rightarrow q$ be a left limit transition and $q^{\prime} \rightarrow P^{\prime}$ be a right limit transition. If $q \in P, q^{\prime} \in P^{\prime}$ and $P=P^{\prime}$, then $q=q^{\prime}$ and for any $R \subseteq P$ with $q \in R$, the left and right transitions $R \rightarrow q$ and $q \rightarrow R$ must appear among the transitions of the automaton.

We do not know a characterization by a particular class of orderings.

7 This class corresponds to the Kleene theorem of [3] for all countable scattered orderings. The operation $\diamond$ is here used as a binary operation.

Let us illustrate by examples some classes of the hierarchy.

Example 4 The set $\left(b^{-\omega} a b^{\omega}\right)^{*}$ of Examples 1 and 2 belong to the Class 2 . The related orderings are $K_{n}=\sum_{j \in\{1, \ldots, n\}} \zeta$ which belong to $U_{2}$. Since the linear ordering $\zeta$ is neither an ordinal nor a backwards ordinal, the set $\left(b^{-\omega} a b^{\omega}\right)^{*}$ cannot belong to a lower class.

Example 5 The automaton of Figure 7 accepts the rational set $(a+b b)^{\diamond}$. The operation $\diamond$ is unary and the automaton satisfies Condition $(\dagger)$. Hence it is 


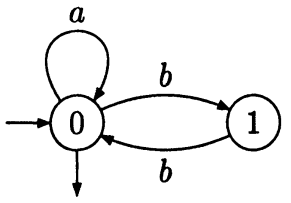

$$
\begin{gathered}
0 \rightarrow\{0\} \\
0 \rightarrow\{0,1\} \\
\{0\} \rightarrow 0 \\
\{0,1\} \rightarrow 0
\end{gathered}
$$

Figure 7. Automaton accepting the set $(a+b b)^{\circ}$

an example of Class 6. Note that it does not belong to Class 5. Indeed, the condition on the limit transitions of the automaton is not respected. Moreover, the set $(a+b b)^{\circ}$ contains the word $\prod_{j \in J} a$ of length $J=\zeta^{\omega}$ and we have seen in Section 2 that $J$ belongs to $W_{\omega}$ but not to $\bigcup_{n<\omega} W_{n}$.

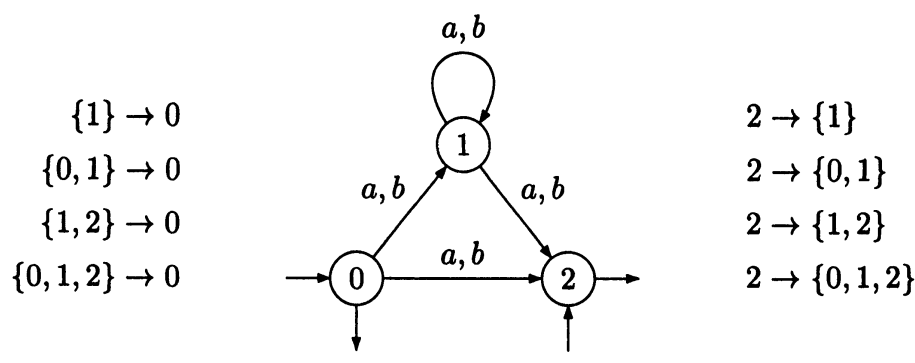

Figure 8. Automaton accepting the set $\varepsilon \diamond A$

Example 6 The automaton pictured in Figure 8 accepts the set denoted by the rational expression $\varepsilon \diamond A$ where $A=\{a, b\}$. Recall that a linear ordering is complete if any subset which is upper bounded has a least upper bound (or equivalently if any subset which is lower bounded has a greatest lower bound). It can be shown that a scattered linear ordering $J$ is complete iff there is a scattered linear ordering $K$ such that $J=\hat{K}$. Therefore, the automaton of Figure 8 accepts words the length of which is a complete ordering.

This automaton does not satisfy Condition $(\dagger)$. Indeed there exist left and right limit transitions $P \rightarrow q$ and $P^{\prime} \rightarrow q^{\prime}$ such that $q \in P, q^{\prime} \in P^{\prime}$ and $P=P^{\prime}$ but $q \neq q^{\prime}$. Take $P=P^{\prime}=\{0,1,2\}, q=2$ and $q^{\prime}=0$. It can be shown that any rational expression with a unary $\diamond$ denotes a set which contains words with a non complete length. Therefore the set $\varepsilon \diamond A$ does not belong to Class 6 .

\section{References}

[1] N. Bedon. Langages reconnaissables de mots indéxés par des ordinaux. Thèse de doctorat, Université de Marne-la-Vallée, 1998. 
[2] N. Bedon and O. Carton. An Eilenberg theorem for words on countable ordinals. In Cláudio L. Lucchesi and Arnaldo V. Moura, editors, Latin'98: Theoretical Informatics, volume 1380 of Lect. Notes in Comput. Sci., pages 53-64. SpringerVerlag, 1998.

[3] V. Bruyère and O. Carton. Automata on linear orderings. In J. Sgall, A. Pultr, and P. Kolman, editors, MFCS'2001, volume 2136 of Lect. Notes in Comput. Sci., pages 236-247, 2001. IGM report 2001-12.

[4] J. R. Büchi. Weak second-order arithmetic and finite automata. Z. Math. Logik und grundl. Math., 6:66-92, 1960.

[5] J. R. Büchi. Transfinite automata recursions and weak second order theory of ordinals. In Proc. Int. Congress Logic, Methodology, and Philosophy of Science, Jerusalem 1964, pages 2-23. North Holland, 1965.

[6] Y. Choueka. Finite automata, definable sets, and regular expressions over $\omega^{n}$ tapes. J. Comput. System Sci., 17(1):81-97, 1978.

[7] D. Girault-Beauquier. Bilimites de langages reconnaissables. Theoret. Comput. Sci., 33(2-3):335-342, 1984.

[8] S. C. Kleene. Representation of events in nerve nets and finite automata. In C.E. Shannon, editor, Automata studies, pages 3-41. Princeton university Press, Princeton, 1956.

[9] R. McNaughton and S. Papert. Counter free automata. MIT Press, Cambridge, MA, 1971.

[10] D. Muller. Infinite sequences and finite machines. In Proc. of Fourth Annual IEEE Symp., editor, Switching Theory and Logical Design, pages 3-16, 1963.

[11] M. Nivat and D. Perrin. Ensembles reconnaissables de mots bi-infinis. In Proceedings of the Fourteenth Annual ACM Symposium on Theory of Computing, pages 47-59, 1982.

[12] D. Perrin. Finite automata. In J. van Leeuwen, editor, Handbook of Theoretical Computer Science, volume B, chapter 1, pages 1-57. Elsevier, 1990.

[13] J. G. Rosenstein. Linear ordering. Academic Press, New York, 1982.

[14] M.-P. Schützenberger. On finite monoids having only trivial subgroups. Inform. Control, 8:190-194, 1965.

[15] W. Thomas. Automata on infinite objects. In J. van Leeuwen, editor, Handbook of Theoretical Computer Science, volume B, chapter 4, pages 133-191. Elsevier, 1990.

[16] J. Wojciechowski. Finite automata on transfinite sequences and regular expressions. Fundamenta informatica, 8(3-4):379-396, 1985. 